\title{
Extraskeletal Myxoid Chondrosarcoma of Leg in A 35 Years Old Female: Report of A Rare Malignant Tumor of Uncertain Differentiation
}

Dr. Rubeena Naz Mohroo, M.D ${ }^{1}$, Dr. Mohammad Jaseem Hassan, M.D ${ }^{2 *}$, Dr. Safia Rana, DCP, DNB ${ }^{3}$, Dr. Sabina Khan, M.D ${ }^{2}$, Dr. Sujata Jetley, M.D ${ }^{4}$

${ }^{1}$ Demonstrator, ${ }^{2}$ Associate Professor, ${ }^{3}$ Assistant Professor, ${ }^{4}$ Professor, Department of Pathology, Hamdard Institute of Medical Sciences and Research (HIMSR), Jamia Hamdard, New Delhi, 110062, India

\author{
DOI: $10.36348 /$ sjpm.2020.v05i06.001 \\ | Received: 21.05.2020 | Accepted: 31.05.2020 | Published: 06.06.2020
}

*Corresponding author: Dr. Mohammad Jaseem Hassan

Abstract

Extraskeletal myxoid chondrosarcoma is a rare malignant soft tissue tumor of uncertain differentiation, constituting less than $3 \%$ of all soft tissue sarcomas. This low grade malignant tumor with high recurrence and metastatic potential, occurs predominantly in proximal extremities and limb girdles of males with thigh being the most common location. Here we present a case in a 35 years old female, who presented with pain and large swelling of around $8 \mathrm{~cm}$ in lateral side of left leg since five months. Wide local excision of the tumor was done and on histopathology a diagnosis of Extraskeletal myxoid chondrosarcoma was rendered. On Immunohistochemistry the tumor cells showed reactivity for Vimentin, S100 and NSE and was non-reactive for CK. Thus our histopathological diagnosis was reconfirmed by immunohistochemistry. Despite high incidence of local recurrence and metastasis, ESMC has an excellent overall survival rate.

Keywords: Extraskeletal, Low grade sarcoma, Malignant potential, Myxoid, Uncertain differentiation.

Copyright @ 2020: This is an open-access article distributed under the terms of the Creative Commons Attribution license which permits unrestricted use, distribution, and reproduction in any medium for non-commercial use (NonCommercial, or CC-BY-NC) provided the original author and sources are credited.

\section{INTRODUCTION}

Soft tissue sarcomas accounts for less than $1 \%$ of the overall burden of malignant tumors in human being [1]. Extraskeletal myxoid chondrosarcoma (ESMC) is a very rare soft tissue tumor, which accounts for less than 3\% of all soft tissue sarcomas [1]. Although first described by Stout and Verner in 1953, it was Enzinger and Shiraki who described ESMC as a distinct clinicopathological entity in 1972 in their study of 34 cases [2, 3]. The 2002 edition of WHO classification of tumors of soft tissue and bones recognised ESMC as a specific entity and categorised it in malignant category of tumor of uncertain differentiation [4]. As per WHO, ESMC is characterised by "a multinodular architecture, abundant myxoid matrix, and malignant chondroblast-like cells arranged in cords, clusters, or delicate networks" [4]. However contrary to its name, no convincing evidence of cartilaginous differentiation is seen in ESMC [5], hence it is different from Extraskeletal mesenchymal chondrosarcoma. ESMC primarily occurs in $5^{\text {th }}$ to $7^{\text {th }}$ decade of life and seen twice as commonly in males than females. Deep soft tissues of the proximal extremities and limb girdles are the most common location for this tumor, however they can also be seen in trunk, paraspinal region, foot, and head and neck region. Intracranial location, fingers, retroperitoneum, pleura and bone is rare site for this tumor [4]. Here we report a case of ESMC in a 35 years old female who presented with swelling and pain in left lower leg since five months.

\section{CASE HISTORY}

A 35 years old female patient presented with swelling and pain in left lower leg since five months. Swelling was present at the lateral aspect of the left leg and appears firm, non-tender, non-mobile, nonpulsatile, about $9 \times 5 \mathrm{~cm}$ in size with normal overlying skin and normal adjacent underlying structure. MRI of the left leg shows well defined encapsulated, lobulated soft tissue mass lesion in the peroneus muscle belly at left mid leg laterally, with no neurovascular or bony involvement. The patient was admitted and wide local excision of the mass was planned. Pre-operative hematological and biochemical tests were within normal limit. The mass was excised and sent for histopathological examination. On gross examination, an encapsulated elongated grey white to grey brown soft to firm mass with nodular external surface measuring $8 \times 4 \times 2.5 \mathrm{~cm}$ was received which on cut section shows multiple gelatinous nodules separated by fibrous septa. (Fig-1A \&1B) Areas of hemorrhage was also seen. Microscopic examination of the mass showed 
an encapsulated multinodular architecture in which tumor cells are arranged in lobules and cords, filled with myxoid or chondromyxoid material and separated by fibrous tissue. (Fig-2A to 2D) Tumor cells were moderately pleomorphic, round to oval in shape with irregular nuclei, open chromatin, prominent nucleoli and eosinophilic to clear cytoplasm. Mitosis was sparse.
Thus based on these features the diagnosis of Extraskeletal myxoid chondrosarcoma (ESMC) was rendered histopathologically. On immunohistochemistry (IHC) the tumor cells showed positivity for Vimentin, S100 and NSE, and was negative for CK. Thus IHC reconfirms our histopathological diagnosis of ESMC.

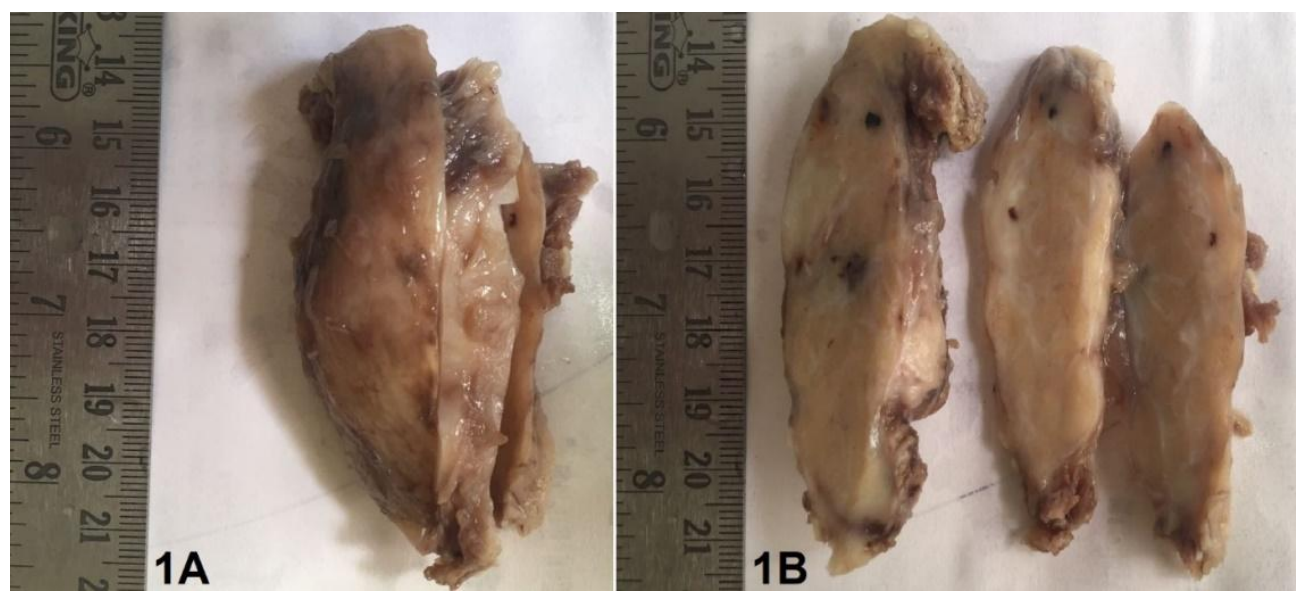

Fig-1: Gross Examination. (1A) External surface showing encapsulated, glistening external surface with nodules of variable size on the surface.

(1B) Cut surface the mass show multiple gelatinous nodules of variable size separated by fibrous septa. Areas of haemorrhage is also seen

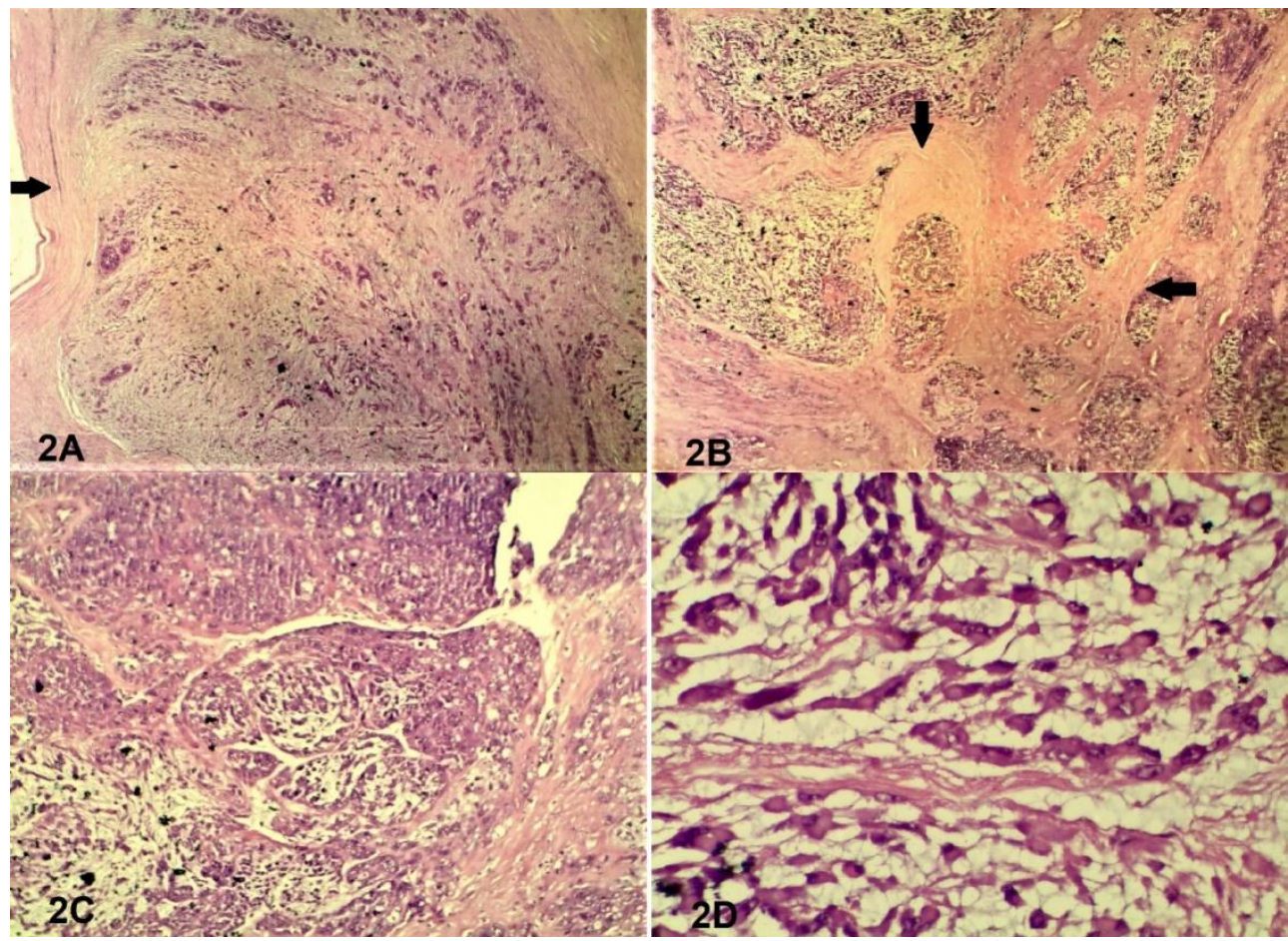

Fig-2: Microscopic Examination. (2A) Low power view showing encapsulated (arrow) circumscribed tumor with tumor cells arranged in cords within large areas of myxoid material. (H\&Ex100) (2B) Scanner view showing multinodular architecture of tumor cells separated by fibrous septa (arrow). (H\&Ex40) (2C) Low power view showing sheets and cords of tumor cells filled with myxoid material within nodule. (H\&Ex100)

(2D) High power view showing tumor cells connect with each other forming cords within the abundant myxoid material. (H\&Ex400)

\section{DISCUSSION}

Extra skeletal myxoid chondrosarcoma is rare, slow growing, low grade malignant soft tissue tumour with high local recurrence and metastatic potential. ESMC occurs mainly in deep soft tissues of the proximal extremities and limb girdles in individuals of 50-70 years of age group [4]. Thigh is the most common location for this tumor, however this tumor can be present in rare locations like head and neck region, trunk, paraspinal region, intracranial regions etc. as well [4]. Our patient was a 35 years old female who presented with pain and swelling of left leg. Sayal NR et al., reported a case of ESMC of neck in a 65 years old male who presented with large neck mass occluding the airway and causing dyspnoea [6]. Zhang L et al. in 
their 12 years of study reported 13 cases of ESMC. In their study in addition to extremities they have also reported one case each in spinal canal, nasopharynx, wall of chest, right lower jaw and right lumbosacral region of the spine [5]. Drilon AD et al., studied the clinical behaviour and treatment responses of 87 cases of ESMC in their retrospective review study over a period of 33 years. In their study out of 87 cases of ESMC, 53 cases were present in lower extremity, 15 cases were seen in upper extremity, 11 cases were reported in abdomen, retroperitoneum and pelvic area, 5 cases were seen in chest wall \& abdominal wall and one case was seen in intrathoracic region and one case was reported from head and neck region [7]. Rao $\mathrm{P}$ et al., reported a case of thoracic intradural ESMC in a 29 years old male patient [8].

Microscopically conventional well differentiated ESMC has characteristic multinodular architecture in which circumscribed tumor cells filled will myxoid/chondromyxoid stroma is separated by fibrous septa [4]. The tumor cells are usually arranged in cords and clusters and have round to oval nuclei with inconspicuous nuclei and moderate amount of eosinophilic granular to vacuolated cytoplasm. The mitotic activity is usually low. Areas of hemorrhage are also seen [4]. Our case show all the features of conventional ESMC. The cellular variant of ESMC is characterised by high cellularity with closely spaced epithelioid cells with minimal myxoid stroma [4]. High grade ESMC is characterised by sheets of anaplastic epithelioid cells with fibrosarcomatous area, high mitotic activity and large areas of necrosis without myxoid/chondromyxoid stroma [4].

ESMC has no distinct diagnostic immunohistochemical profile. However vimentin is generally expressed in $100 \%$ of cases [4], Immunoreactivity for Neuron- specific enolase is seen in $100 \%$ cases, Synaptophysin in $87 \%$ cases, S100 in $50 \%$ cases and Epithelial membrane antigen in $25 \%$ of cases [9]. Our case showed positivity for Vimentin, S100 and NSE.

Ultrastructural study of ESMC by Hisaoka M et al., confirms the neuroendocrine differentiation of ESMC. They demonstrated neuron-specific microtubule-related proteins MAP-2 and class III tubulin in ESMC [10]. Goh YW et al., also confirms the neuroendocrine differentiation of ESMC by immunophenotypic and ultrastructural study [9]. Both these studies confirms that ESMC is a specific entity and ruling out the possibility of chondrocytic or prechondrocytic origin of ESMC $[9,10]$.

Ancillary cytogenetic and molecular studies can be extremely useful in establishing a definitive diagnosis for ESMC. ESMC is marked by a reciprocal translocation $\mathrm{t} \quad(9: 22) \quad(\mathrm{q} 22 ; \mathrm{q} 12)$ generating a EWS/NR4A3 gene fusion, in approximately $50 \%$ of cases $[4,7]$. This translocations result in fusion gene products, which leads to tumorigenesis by causing alterations in cellular growth and differentiation [7].

ESMC was graded as grade 2/3 tumor by the French Federation of Cancer Centres (FNCLCC) [7]. Treatment of ESMC consist of wide local excision for localised disease, while systemic chemotherapy is recommended for metastatic disease. Despite surgical intervention, a high rate of local recurrence in the range of $14-64 \%$ is reported by various authors [7], while metastatic disease is reported in upto $46 \%$ of cases [8]. Lung is the most common site for metastasis, while extrapulmonary metastasis most commonly reported in soft tissues, lymph nodes and bones [8]. Despite high incidence of local recurrence and metastasis, excellent overall survival for ESMC is reported by various authors. The 5 year survival rate was reported between $82-100 \%[7,11,12], 10$ year survival rate was reported between $65-88 \%$ [7, 11, 12], and 15 years survival rate was reported between $58-60 \%$ [7, 11]. Unfavourable prognostic factors include male sex, older age $(>50$ years), large tumor size $(\geq 10 \mathrm{~cm})$, deep location of the tumor, proximal tumor site, inadequate initial surgery, cellular tumor, presence of anaplasia, high mitotic count and high Ki-67 expression $[11,13]$.

\section{CONCLUSION}

ESMC is a very rare low grade malignant soft tissue tumor, which is recognised as a specific entity and categorised in malignant category of tumor of uncertain differentiation by WHO. Surgical intervention in the form of WLE is the only satisfactory cure of localised tumor, which reduces the chance of local disease recurrence and thus improved the overall survival of the patient. Despite the high local recurrence rate and metastatic potential, ESMC has an excellent overall survival rate with 10 year survival rate in the range of $65-88 \%$.

\section{REFERENCES}

1. Fletcher, C. D. M., Rydholm, A., Singer, S., Sundaram, M., \& Coindre, J. M. (2005). Soft tissue tumours: epidemiology, clinical features, histopathological typing and grading. Fletcher CDM, Unni KK, Mertens F, editors. World health organization classification of soft tissue tumours. Lyon (France): IARC Press, 10-223.

2. Stout, A. P., \& Verner, E. W. (1953). Chondrosakcoma of the extraskeletal soft tissues. Cancer, 6(3), 581-590.

3. Enzinger, F. M., \& Shiraki, M. (1972). Extraskeletal myxoid chondrosarcoma: an analysis of 34 cases. Human pathology, 3(3), 421-435.

4. Fletcher, C., Unni, K., \& Mertens, F. (2002). Extraskeletal myxoid chondrosarcoma. Lucas DR, Heim S. World Health Organization Classification of Tumours: pathology and genetics of tumours of soft tissue and bone. Lyon: IARCPress, 213-215. 
5. Zhang, L., Wang, R., Xu, R., Qin, G., \& Yang, L. (2018). Extraskeletal myxoid chondrosarcoma: a comparative study of imaging and pathology. BioMed research international, 2018.

6. Sayal, N. R., Lepoudre, C., \& Pui, J. (2017). Extraskeletal myxoid chondrosarcoma of the neck. Otolaryngology Case Reports, 2, 22-25.

7. Drilon, A. D., Popat, S., Bhuchar, G., D'Adamo, D. R., Keohan, M. L., Fisher, C., ... \& Maki, R. G. (2008). Extraskeletal myxoid chondrosarcoma: a retrospective review from 2 referral centers emphasizing long- term outcomes with surgery and chemotherapy. Cancer, 113(12), 3364-3371.

8. Rao, P., Colen, R. R., Bruner, J. M., \& Meis, J. M. (2014). Extraskeletal myxoid chondrosarcoma presenting as an intradural spinal mass: Report of a rare clinical presentation with an emphasis on differential diagnostic considerations. Rare tumors, 6(4), 150-153.

9. Goh, Y. W., Spagnolo, D. V., Platten, M., Caterina, P., Fisher, C., Oliveira, A. M., \& Nascimento, A. G. (2001). Extraskeletal myxoid chondrosarcoma: a light microscopic, immunohistochemical, ultrastructural and immuno- ultrastructural study indicating neuroendocrine differentiation. Histopathology, 39(5), 514-524.
10. Hisaoka, M., Okamoto, S., Koyama, S., Ishida, T., Imamura, T., Kanda, H., ... \& Hashimoto, H. (2003). Microtubule-associated protein-2 and class III $\beta$-tubulin are expressed in extraskeletal myxoid chondrosarcoma. Modern pathology, 16(5), 453 459.

11. Meis-Kindblom, J. M., Bergh, P., Gunterberg, B., \& Kindblom, L. G. (1999). Extraskeletal myxoid chondrosarcoma: a reappraisal of its morphologic spectrum and prognostic factors based on 117 cases. The American journal of surgical pathology, 23(6), 636-650.

12. Kawaguchi, S., Wada, T., Nagoya, S., Ikeda, T., Isu, K., Yamashiro, K., ... \& Matsumoto, S. (2003). Extraskeletal myxoid chondrosarcoma: a multi- institutional study of 42 cases in Japan. Cancer: Interdisciplinary International Journal of the American Cancer Society, 97(5), 1285-1292.

13. Oliveira, A. M., Sebo, T. J., McGrory, J. E., Gaffey, T. A., Rock, M. G., \& Nascimento, A. G. (2000). Extraskeletal myxoid chondrosarcoma: a clinicopathologic, immunohistochemical, and ploidy analysis of 23 cases. Modern Pathology, 13(8), 900-908. 